\title{
Experimental Observation of the Crystallization of Hard-Sphere Colloidal Particles by Sedimentation onto Flat and Patterned Surfaces
}

\section{Citation}

Ramsteiner, I.B., Katherine Estelle Jensen, David A. Weitz, and Frans A. Spaepen. 2009.

Experimental observation of the crystallization of hard-sphere colloidal particles by sedimentation onto flat and patterned surfaces. Physical Review E 79(011403).

\section{Published Version}

doi:10.1103/PhysRevE.79.011403

\section{Permanent link}

http://nrs.harvard.edu/urn-3:HUL.InstRepos:4262332

\section{Terms of Use}

This article was downloaded from Harvard University's DASH repository, and is made available under the terms and conditions applicable to Open Access Policy Articles, as set forth at http:// nrs.harvard.edu/urn-3:HUL.InstRepos:dash.current.terms-of-use\#OAP

\section{Share Your Story}

The Harvard community has made this article openly available.

Please share how this access benefits you. Submit a story.

\section{Accessibility}




\title{
Experimental Observation of the Crystallization of Hard Sphere Colloidal Particles by Sedimentation onto Flat and Patterned Surfaces
}

\author{
I.B. Ramsteiner, K.E. Jensen, D.A. Weitz, and F. Spaepen \\ Harvard University, School of Engineering and Applied Sciences, \\ 9 Oxford Street, Cambridge, Massachusetts 02138, USA
}

(Dated: December 13, 2008)

\begin{abstract}
We present a confocal microscopy study of $1.55 \mu \mathrm{m}$ monodisperse silica hard spheres as they sediment and crystallize at the bottom wall of a container. If the particles sediment onto a featureless flat wall, the two bottom layers crystallize simultaneously and layerwise growth follows. If the wall is replaced by a hexagonal template, only layerwise growth occurs. Our results complement earlier numerical simulations and experiments on other colloidal systems.

PACS numbers: Valid PACS appear here
\end{abstract}




\section{INTRODUCTION}

Phase transformations in colloidal hard spheres have been fascinating physicists for more than half a century.[1, 2] That purely entropic effects can produce a first-order crystallization transition is particularly fascinating. [3] The bulk phase diagram of monodisperse hard spheres has been verified experimentally and is now widely accepted.[4] The vast increase in studies on the particle level has been driven by the rapid development of two technologies: confocal microscopy and fast processing of large amounts of data. Thus, state-of-the-art simulations of typically $10^{5}$ particles can be directly compared to real colloidal suspensions. [5, 6] Their value to the field of statistical mechanics derives from the variety of interaction mechanisms (Coulomb, van der Waals, depletion and steric interactions) that make it possible to tune independently the range and strength of particle interactions.[7-9] A particularly exciting development is the use of monodisperse hard sphere colloids to simulate structural dynamics in dense atomic or molecular systems,[10] phenomena that are experimentally inaccessible on the atomic scale. Examples include homogeneous and heterogeneous nucleation of crystals,[11, 12] crystal-fluid interfaces,[13] glass formation,[14, 15] matter under shear $[16,17]$ or thermal capillary waves.[18] Hard sphere crystals are excellent models for simple metals. [19, 20] They are also of interest as photonic bandgap materials.[21, 22]

Colloidal crystals can be grown in several ways.[23-27] A simple, widely used method is the sedimentation of particles from a dilute suspension.[28-31] A hard sphere suspension in equilibrium in a gravitational field usually consists of four regions from top to bottom: a clear fluid, a uniform dispersion, the so-called fan in which the density increases smoothly with depth, and a compact sediment.[28] Unless the sedimentation occurs too fast and the particles jam into a glassy state, the sediment of a monodisperse hard sphere suspension is crystalline. The precrystallization of fluids at patterned surfaces [34, 35] allows the fabrication of large single crystals with various orientations by directing the growth with a template.[36] Such templates are structural patterns at the bottom of the container that act as crystallization seed. Template-directed sedimentation is a very popular method for the production of large colloidal crystals for scientific purposes. In the future it may well allow the cheap, large scale fabrication of photonic crystals.

Several aspects of crystallization by sedimentation are still poorly understood. Questions remain not only about dynamics, but also about the equilibrium states. These questions have 
been addressed by a number of experiments and computer simulations: x-ray attenuation experiments, [28] particle-scale studies by confocal microscopy,[31, 32] DFT calculations, [33] simulations of a fixed number of hard sphere particles in an increasing gravitational field,[29] and, most recently, a grand canonical Monte Carlo study that incorporated experimentally more accessible conditions (constant gravitation). Particularily interesting are the early stages of sedimentation and the crystallization of the very first layers. Previous results suggest that at a flat bottom wall two or more layers (depending on the gravitational length, which is introduced below) crystallize simultaneously, while further crystal growth proceeds layer by layer. This is not a kinetic effect, but one associated with the hard sphere system in equilibrium. [37] It is not only important from an academic point of view, concerning the ever fascinating subject of phase transitions. It is also interesting in view of future efforts to optimize quality and production efficiency of self-organizing photonic crystals.

In this paper we investigate experimentally the early sedimentation stages of colloidal silica hard spheres onto a flat bottom wall. We select parameters for which we expect from earlier simulation work the simultaneous crystallization of two layers. Furthermore, we are interested in which way a template affects this phenomenon. The colloids are imaged with laser confocal scanning microscopy and the data evaluated on the particle level.

\section{EXPERIMENTAL DETAILS}

We approximate an ideal hard sphere system using a suspension of spherical silica particles in a fluorescein-dyed solution of dimethyl sulfoxide (DMSO) and water. The silica particles (Micromod Sicastar) have a diameter of $\sigma=1.55 \mu \mathrm{m}$ and a polydispersity of less than $3.5 \%$ [19]. The aqueous stock suspension (volume fraction 0.025) is homogenized with a vortexer, sonicated for at least 5 minutes, and allowed partially to settle. At infinite dilution we expect for the particles a sedimentation velocity of $u_{0}=\frac{1}{18} \sigma^{2} \Delta \rho g / \eta \approx 4.7 \mathrm{~mm} /$ hour, where $\Delta \rho=1 \mathrm{~g} / \mathrm{cm}^{3}$ is the density difference between the particles and the liquid, $g=9.81 \mathrm{~m} / \mathrm{s}^{2}$ the gravitational constant and $\eta=10^{-3} \mathrm{~Pa}$ s the viscosity of water. In agreement with this estimate we observe distinct layering of the sample after 6 hours. At this time, there is a roughly 3 -cm-thick region of clear water at the top followed by a sharp transition to a milky suspension below and a small, compact sediment beginning to form at the bottom of the vial. Since dumbbells and larger particle clusters sediment at least twice as fast as single 
particles, we avoid these large impurities by drawing only from the top $3 \mathrm{~cm}$ of the milky suspension. We extract a small amount with a pipette and prepare a suspension with a particle volume fraction of $1.3 * 10^{-4}$ [44] in $62.8 \%$ (by volume) DMSO and $37.2 \%$ water. This solution is designed to match the particles' index of refraction in order to minimize van der Waals forces and permit imaging deep into the sample with minimal scattering. Under these conditions, the particles have the inverse gravitational length $g^{*}=m^{*} g \sigma / k_{B} T \approx 7$ where $m^{*}=\left(\frac{1}{3} \pi \sigma^{2}\right) \Delta \rho$ is the relative particle mass. Additionally, we add a small amount of fluorescein- $\mathrm{NaOH}$ for fluorescence microscopy.

Our sample cell consists of a glass tube with an inner diameter of $5 \mathrm{~mm}$ affixed with UV-hardened glass glue to a microscope coverslip. Once the glue is fully hardened, we rinse the cell with water followed by a water/DMSO solution, then fill it with the dyed, index-matched suspension and seal it with Parafilm. We mount the sample on a Leica TCS SP5 point scanning confocal microscope, taking care to check the alignment of the sample stage with respect to the direction of gravity using a bulls-eye water level. We image the sample from below using a 100X oil-immersion objective by fluorescence microscopy. The microscope laser outputs $488 \mathrm{~nm}$ light to excite the fluorescein dye, then collects and selectively detects fluorescence from the focal point. Rastering mirrors allow point scanning in the $\mathrm{x}-\mathrm{y}$ plane (orthogonal to gravity), while changing the focus enables us to image at different depths in the sample. Data from a single time step consists of a stack of $x-y$ images taken over a desired range in the z-direction. In this way, we obtain $3 \mathrm{D} 512 \times 512 \times 80$ voxel data, corresponding to a sample volume of $93 \times 93 \times 16 \mu \mathrm{m}$. Scanning one stack of 80 images takes $13.5 \mathrm{~s}$. The silica particles appear dark in the dyed liquid.

The raw data is processed using an IDL software package based on algorithms by Crocker and Grier [38, 39]. Noise and spatial intensity variations are removed by a bandpass filter. Stationary particles are located with an accuracy of $0.1 \sigma$ or better. Isolated, fast-moving particles are smeared out due to the limited speed of scanning in the z-direction. This presents no problem for the current work, however, since exact coordinates are only needed for the relatively immobile, crystallizing particles.

This work focuses on crystallization at a flat surface, but for comparison we also report the experiment of crystal formation on a patterned substrate. Our template is a hexagonal pattern of holes that mimicks the bottom layer of a [111]-oriented single crystal. The pattern and pitch $(1.65 \mu \mathrm{m})$ of the holes is chosen to match that of the naturally-forming crystal on a 
flat surface. We produce the pattern by using reactive ion etching and standard lithography techniques from a $\sigma / 2$-thick PMMA film spun onto a microscope coverslip and then construct the sample cell as described above.

\section{RESULTS AND DISCUSSION}

We image the suspension near to the bottom wall of the sample cell every six minutes. Over the course of 13 hours we observe the formation of a sediment with an area density of ca. $6 / \sigma^{2}$ in the final measurement. Complete sedimentation of all particles would take about twice that time. Thus, a small but constant flux of particles is maintained during the entire course of the experiment.

From the particle positions we calculate the density profile

$$
\rho(z)=\frac{1}{A \Delta z} \iiint_{z}^{z+\Delta z} d x d y d z \rho(x, y, z)
$$

by counting particles in $\Delta z=0.1 \mu \mathrm{m}$ thick slabs. $A$ is the lateral $\mathrm{x}-\mathrm{y}$ area of the sampled region. Density profiles at different sedimentation stages are shown in Fig. 1. The individual curves are labeled with the area density - the total number of particles in the observed volume at that stage divided by the area A. This quantity is close to the area density of the sediment, since the homogeneous suspension is extremely dilute. From the very beginning we observe a pronounced layering. As the layers crystallize, the corresponding peaks become narrower and higher, while the minima between them eventually drop to zero. Their actual depth relative to the corresponding bulk density has been suggested as a simple and robust crystallization criterion.[35] The arbitrariness of the threshold (for example 5\%), however, is somewhat unsatisfactory. In fact, it has been shown that even for simultaneously crystallizing layers the respective local densities drop below $5 \%$ of the bulk density at significantly different stages of the sedimentation.[31]

More reliable and precise crystallization criteria assign to every single particle or interparticle bond an individual order parameter based on the arrangement of its neighbors.[40, 41] Following the authors of a recent simulation work [37], we introduce the order parameter

$$
\psi_{j}^{(6)}=\frac{1}{N_{j}} \sum_{k=1}^{N_{j}} e^{i 6 \theta_{j k}}
$$

with the sum over all $k=\left\{1 \ldots N_{j}\right\}$ neighbors of particle $j . \theta_{j k}$ is the angle between $\mathbf{r}_{j k}=$ $\mathbf{r}_{j}-\mathbf{r}_{k}$ and an arbitrary reference direction in the $\mathrm{x}-\mathrm{y}$ plane. Neighbors of $j$ are particles 


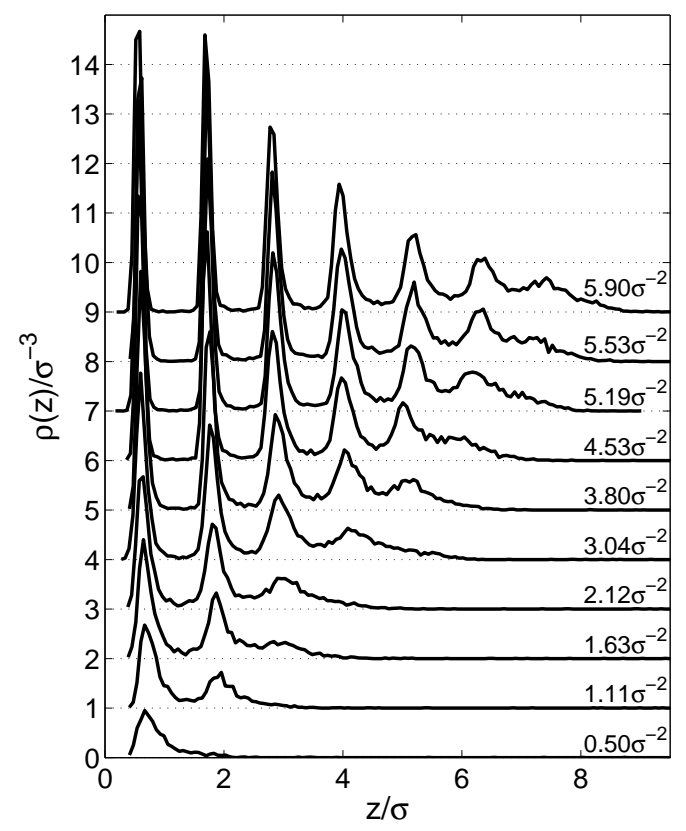

FIG. 1: Density profiles at various stages of sedimentation. The plots are shifted vertically and labeled with the respective area density (see text).

with $\left|\mathbf{r}_{j k}\right|<1.3 a_{0}$, based on the experimentally observed nearest neighbor distance $a_{0}$. For the identification of neighbors the $z$-coordinates are multiplied by a factor of 4 in order to ensure neighbor selection in the same layer. For each particle $\psi_{j}^{(6)}$ is a complex number with $0 \leq\left|\psi_{j}^{(6)}\right| \leq 1$. For particles embedded in a crystalline layer the argument of $\psi_{j}^{(6)}$ depends on the local crystal orientation.

Fig. 2 shows snapshots of the first three layers at four different crystallization stages. The images are $\mathrm{x}-\mathrm{y}$ projections of all particles within $0.65 \sigma$ intervals around the layer positions (the peaks in Fig. 1).[45] $\left|\psi_{j}^{(6)}\right|$ for each particle is indicated by the greyscale, with darker shades indicating more crystalline environments. It is apparent that crystallization proceeds by nucleation and growth. For the first two layers, this seems to happen roughly at the same time, while the third layer crystallizes later.

For a more quantitative study of the layerwise crystallization we introduce a z-dependent order parameter

$$
\psi^{(6)}(z)=\left\langle\left|\psi_{j}^{(6)}\right|\right\rangle_{\Delta z}
$$




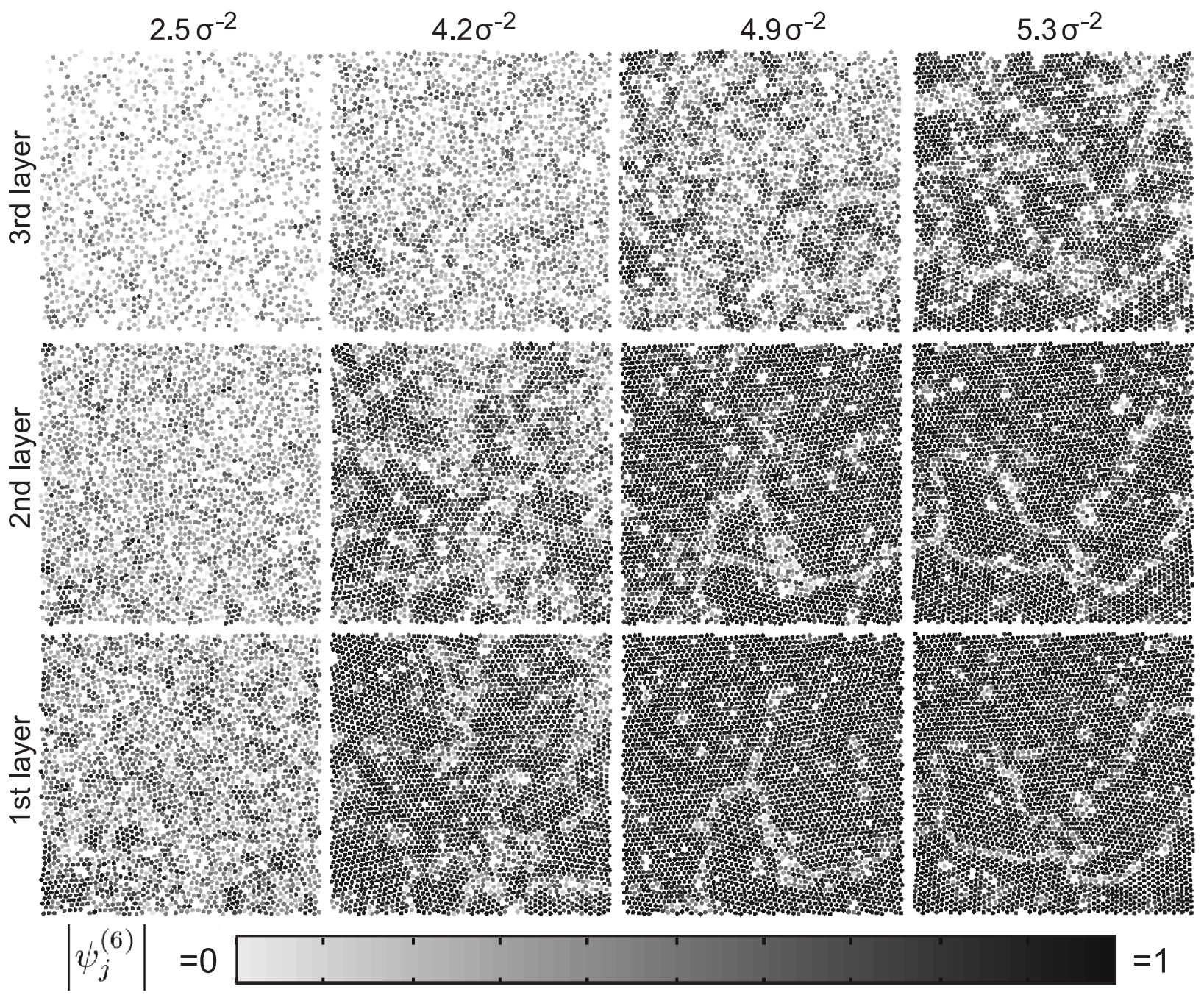

FIG. 2: Selected snapshots of the first three sedimented layers for four different area densities (labels on top). Darker particles have higher order parameters.

which averages the absolute value of $\psi_{j}^{(6)}$ of all particles within $z \pm \frac{\Delta z}{2}$. Several other publications employ the modulus of the complex mean value $\left|\left\langle\psi_{j}^{(6)}\right\rangle\right|$. For a single crystal, that method has the merit of changing from zero (disorder) to unity (perfect crystal) and is therefore useful for systems in which grain boundaries are suppressed, for example by a template or by periodic boundary conditions. [37] For a polycrystal, however, the orientationsensitive arguments of the individual $\psi_{j}^{(6)}$ can produce a very small value for $\left|\left\langle\psi_{j}^{(6)}\right\rangle\right|$, even if the grains are fully ordered. Since the final state in our experiment is a polycrystal, we use the definition given in Eq. 3. When comparing our results to those of others it is crucial to be aware of that difference.

From the particle density profiles (Fig. 1) we determine the location of the layers in $z$. 

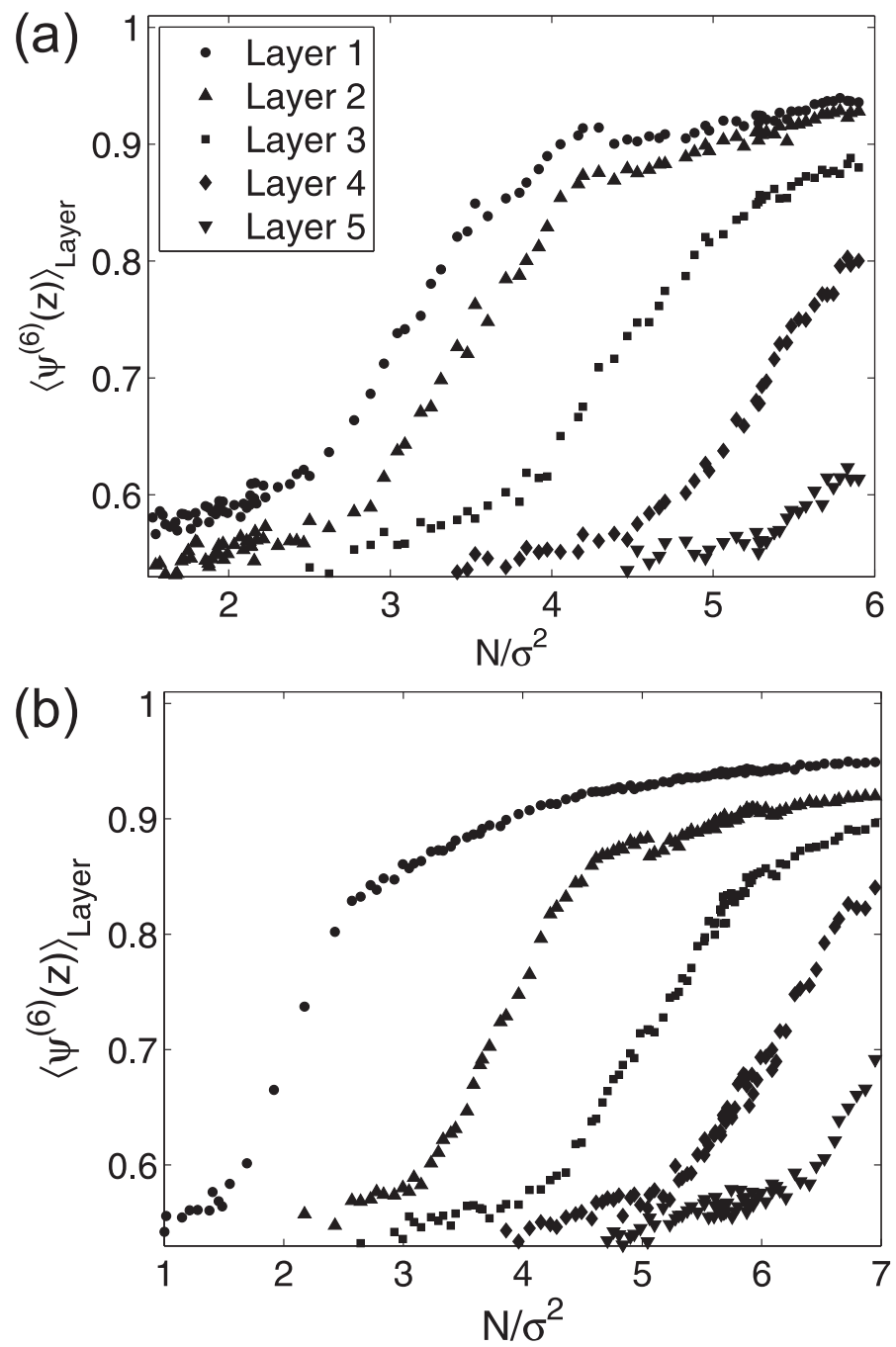

FIG. 3: Mean order parameter as a function of the projected particle density (see text) for the first five layers sedimenting (a) onto a flat surface and (b) onto a [111] template.

For each layer the mean order parameter $\left\langle\psi^{(6)}(z)\right\rangle_{\text {Layer }}$ is calculated by averaging $\psi^{(6)}(z)$ over the $z$-interval defined by the two minima on either side of the respective peak in the density profile.

In Fig. 3a this quantity is shown for the first five layers as a function of the total area density. Each curve consists of three stages that can be understood by comparison with Fig. 2: The first (not shown) is an increase in order parameter from zero to a plateau at 0.55 ( 0.6 for the first layer). This represents the overall increase in density, since $\psi_{j}^{(6)}$ for particles without any close neighbors is zero. The second stage is the crystallization, during which the order parameter steeply rises to $0.85-0.9$ followed by a third stage, in which the 
increase is much slower and represents the annihilation of point defects and grain coarsening. The two discontinuities in the slope of each curve allow exact identification of the onset and completion of crystallization. Therefore, we can confirm what was already qualitatively observed in the snapshots in Fig. 2: The first two layers crystallize simultaneously, and the higher layers individually at regular intervals. One should not be bothered by $\left|\left\langle\psi_{j}^{(6)}\right\rangle\right|$ always being a little smaller in the second layer than in the first. Naturally, the defect concentration has to be higher in the second layer, since artifacts in the first layer (such as grain boundaries) propagate into the second layer or impede its formation. The essential observation is that discontinuities occur simultaneously. Crystallization of the third layer sets in around $4 \sigma^{-2}$, exactly when it is finished in the first two. Therefore, the third layer clearly crystallizes later.

These findings are in general agreement with recent hard sphere simulations and experiments with charged colloids.[31, 37] In Marechal and Dijkstra's report, however, a strongly discontinuous jump in the order parameter when the first two layers crystallize, while the parameter increases more continuously for subsequent layers. Our results resemble more those of Hoogenboom et al. with charge-stabilized colloids: we do not observe a significantly different slope for the third and higher curves. Most likely, this discrepancy has its origin in the order parameter $\left|\left\langle\psi_{j}^{(6)}\right\rangle\right|$ used in the simulations, which does not clearly distinguish between the liquid-like and the polycrystalline. A sharp jump is expected when several randomly oriented nuclei form a single crystal plane. When layers grow on top of that single crystal, the hexagonal clusters have a preferred orientation from the beginning. Their growth can be resolved by $\left|\left\langle\psi_{j}^{(6)}\right\rangle\right|$, which increases more gradually in that case.

Fig. 4(a) shows a snapshot of the first two layers when they are both half-crystallized (compare to Fig. 3). Shown are only particles with $\psi_{j}^{(6)}>0.8$ and with at least three neighbors that have the same property. $\left\langle\psi^{(6)}(z)\right\rangle=0.79$ for the first layer (black) and 0.7 for the second (white). It is apparent that shape, size and position of the crystalline grains are strongly correlated between the layers. The grains are a little smaller in the second layer than in the first, as reflected by the minor difference in $\left\langle\psi^{(6)}(z)\right\rangle$, but their nucleation and growth clearly occurs simultaneously in both layers. Fig. 4(b) illustrates the second and third layers at a later stage with $\left\langle\psi^{(6)}(z)\right\rangle=0.7$ in the third layer (white) and 0.87 in the second, which is now the lower one (black). The upper (third) layer is now at exactly the same stage of crystallization as the upper (second) layer in (a), and has the same $\left\langle\psi^{(6)}(z)\right\rangle$. Comparison 

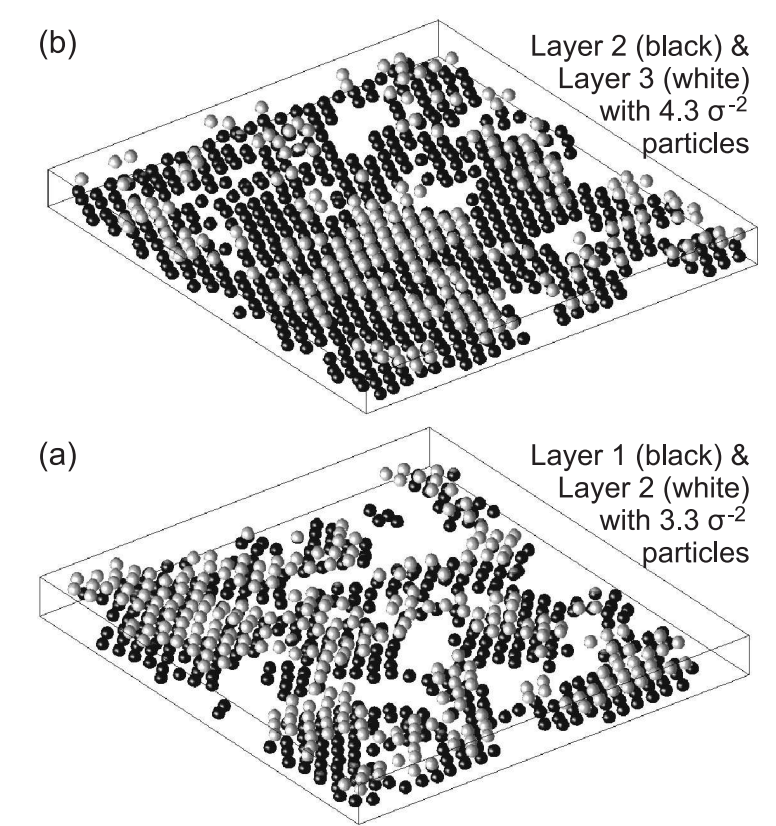

FIG. 4: (a) Graphic reconstruction of the first two layers when $\left\langle\left|\psi^{(6)}(z)\right|\right\rangle=0.7$ in the second layer. (b) the second and third layer at a later time when $\left\langle\left|\psi^{(6)}(z)\right|\right\rangle=0.7$ in the third layer. Only particles with $\left|\psi_{j}^{(6)}\right|>0.8$ are shown.

of the upper layers in (a) and (b) shows similar sizes and shapes of crystalline patches. The respective bottom layers, however, differ substantially: in (a) the first layer is patchy and correlated to the second, while in (b) it covers more area and seems to be almost complete. As before, we can confirm that the first two layers in (a) crystallize simultaneously while (b) resembles epitaxial growth of a layer on a crystal surface.

Fig. 3b shows the results of a sedimentation experiment on a hexagonally patterned template, with hole spacing equal to that of the inter particle spacing on the flat surface. The data were evaluated exactly as for the flat surface. The difference with Fig. 3a is apparent; while the second, third and higher layers crystallize at roughly the same sedimentation stages as before, the first layer does so much earlier. There is clearly no longer simultaneous crystallization of the first two layers.

\section{CONCLUSIONS}

We have studied the sedimentation of spherical silica colloids with an approximately hard sphere interaction potential and a very small gravitational length of $g^{*}=7$ onto a flat 
bottom wall and onto a patterned substrate or template.

At a flat wall the first two layers crystallize simultaneously when the particle density in the sediment increases, while the next layers crystallize individually. Our results generally support recent simulations on the hard sphere system.[37] Differences in detail are most likely the result of a different choice of order parameter in the simulation work. Our aim was to approximate the ideal hard sphere system. Similar experiments in the past used charge stabilized colloidal particles.[31]

For sedimentation onto a template, we observe layerwise crystallization from the beginning. The simultaneous crystallization of the first two layers found in the experiment without a template is therefore clearly induced by the flat wall. Once the second crystal layer is complete, both systems - with or without template - behave similarly.

The phase behavior of hard spheres - particles interacting in the simplest manner imaginable - is of great interest. Understanding the crystallization of a sediment in a gravitational field as thoroughly as possible, however, is not just an exercise in statistical physics. It is a crucial step towards the understanding of real colloidal systems, which almost always are subject to gravity in one way or the other.[42, 43] Furthermore, the sedimentation and crystallization of colloidal particles concerns one of the most exciting potential applications of these systems: cheap and efficient large scale fabrication of photonic crystals. Subtle effects associated with the crystallization of a sediment may be exploited to induce defect structures or gradients by self organization.

Finally, this work provides an excellent example how a field can benefit from the constant interplay between experiments and computer simulations. The number of particles in our sampled volume (up to 20,000) may be matched in some state-of-the-art simulations. The volume we investigate, however, covers only a small part of a much larger real system and is therefore not subject to periodic boundary conditions or to effects from discretization or confinement. Simulations on the other hand represent truly ideal systems and can easily explore a larger parameter space.

This work was supported by NSF (DMR-0602684) and the Harvard MRSEC (DMR0213805).

[1] W. Wood and J. Jacobson, J. Chem. Phys. 27, 1207 (1957). 
[2] B. Alder and T. Wainwright, J. Chem. Phys. 27, 1208 (1957).

[3] W. Hoover and F. Ree, J. Chem. Phys. 49, 3609 (1968).

[4] P. Pusey and W. van Megen, Nature 320, 340 (1986).

[5] H. Yoshida, K. Ito, and N. Ise, Phys. Rev. B 44, 435 (1991).

[6] A. van Blaaderen and P. Wiltzius, Science 270, 1177 (1995).

[7] W. Russel, D. Saville, and W. Schowalter, Colloidal Dispersions (Cambridge Press, 1989).

[8] R. Jones, Soft Condensed Matter (Oxford University Press, 2002).

[9] G. Gompper and M. Schick, Colloidal Order: Entropic and Surface Forces, vol. 3 of Soft Matter (Wiley, 2007).

[10] V. Prasad, D. Semwogerere, and E. Weeks, J. Phys.: Condens. Matter 19, 113102 (2007).

[11] U. Gasser, E. Weeks, A. Schofield, P. Pusey, and D. Weitz, Science 292, 258 (2001).

[12] A. Cacciuto, S. Auer, and D. Frenkel, Nature 428, 404 (2004).

[13] R. Dulles, D. Aarts, and W. Kegel, Phys. Rev. Lett. 97, 228301 (2006).

[14] W. Kegel and A. V. Blaaderen, Science 287, 290 (2000).

[15] E. Weeks, J. Crocker, A. Levitt, A. Schofield, and D. Weitz, Science 287, 627 (2000).

[16] D. Derks, H. Wisman, A. van Blaaderen, and A. Imhof, J. Phys.: Cond. Matt. 16, S3917 (2004).

[17] T. Solomon and M. Solomon, J. Chem. Phys. 124, 134905 (2006).

[18] D. Aarts, M. Schmidt, and H. Lekkerkerker, Science 304, 847 (2004).

[19] P. Schall, I. Cohen, D. Weitz, and F.Spaepen, Science 305, 1944 (2004).

[20] P. Schall, I. Cohen, D. Weitz, and F.Spaepen, Nature 440, 319 (2006).

[21] E. Yablonovich, Phys. Rev. Lett. 58, 2059 (1987).

[22] D. Prather, S. Shi, J. Murakowski, G. Schneider, A. Sharkawy, C. Chen, and B. Miao, IEEE J. Sel. Topics Quantum Electron. 12, 1416 (2006).

[23] N. Denkov, O. Velev, P. Kralchevsky, I. Ivanov, H. Yoshimura, and K. Nagayama, Nature 361, 26 (1993).

[24] S. Park, D. Qin, and Y. Xia, Adv. Mater. 10, 1028 (1998).

[25] P. Jiang, J. Bertone, K. Hwang, and V. Colvin, Che. Mater. 11, 2132 (1999).

[26] L. Meng, H. Wei, A. Nagel, B. Wiley, L. Scriven, and D. Norris, Nano Lett. 6, 2249 (2006).

[27] Z. Cheng, W. Russel, and P. Chaikin, Nature 401, 893 (1999).

[28] K. Davis, W. Russel, and W. Glantschnig, Science 245, 507 (1989). 
[29] T. Biben, R. Ohnesorge, and H. Löwen, Europhysics Letters 28, 665 (1994).

[30] J. Hoogenboom, D. Derks, P. Vergeer, and A. van Blaaderen, J. Chem. Phys. 117, 11320 (2002).

[31] J. Hoogenboom, P. Vergeer, and A. v. Blaaderen, J. Chem. Phys. 119, 3371 (2003).

[32] R. Beckham and M. Bevan, J. Chem. Phys. 127, 164708 (2007).

[33] H. Cheng and H. Ma, J. Chem. Phys. 125, 024510 (2006).

[34] M. Heni and H. Löwen, Phys. Rev. Lett. 85, 3668 (2000).

[35] M. Heni and H. Löwen, J. Phys.: Condens. Matter 13, 4675 (2001).

[36] A. V. Blaaderen, R. Ruel, and P. Wiltzius, Nature 385, 321 (1997).

[37] M. Marechal and M. Dijkstra, Phys. Rev. E 75, 061404 (2007).

[38] http://www.physics.emory.edu/ weeks/idl/.

[39] J. Crocker and D. Grier, J. Colloid Interface Sci. 179, 298 (1996).

[40] P. Steinhardt, D. Nelson, and M. Ronchetti, Phys. Rev. B 28, 784 (1983).

[41] J. Honeycutt and H. Andersen, J. Phys. Chem. 91, 4950 (1987).

[42] J. Zhu, M. Li, R. Rogers, W. Meyer, STS-73 Space Shuttle Crew, W. Russel, and P. Chaikin, Nature 387, 883 (1997).

[43] Z. Cheng, P. Chaikin, J. Zhu, W. Russel, and W. Meyer, Phys. Rev. Lett. 88, 015501 (2002).

[44] Although the exact volume fraction of the extremely dilute homogeneous suspension is difficult to control, it is easily obtained for a given sample from the flux of particles into the sediment.

[45] The numerical value of the interval width is an ad-hoc choice based on the width of the peaks in the $z$ profile. 